INPLASY

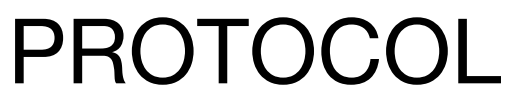

To cite: Zhang et al.

Association between nonalcoholic fatty liver and acute cerebral infarction: a protocol of systematic review and meta-analysis. Inplasy protocol 202040102. doi:

10.37766/inplasy2020.4.0102

Received: 17 April 2020

Published: 17 April 2020

Corresponding author:

Wen-juan Liu

Wen-juanLiu@outlook.com

Author Affiliation:

First Affiliated Hospital of Jiamusi University

Support: HLJPHFPCSRP (2018141)

Review Stage at time of this submission: The review has not yet started.

Conflicts of interest: No.

\section{Association between non-alcoholic fatty liver and acute cerebral infarction: a protocol of systematic review and meta-analysis}

Zhang YJ; Liu WJ².

Review question / Objective: Is non-alcoholic fatty liver (NAFL) associated with acute cerebral infarction ( $\mathrm{ACl})$ ?

Condition being studied: Non-alcoholic fatty liver; acute cerebral infarction.

Information sources: Electronic searches This study will comprehensively search electronic databases (MEDLINE, EMBASE, Cochrane Library, Scopus, Web of Science, WANGFANG, and China National Knowledge Infrastructure) from their sources to the March 1, 2020 without limitations related to the language and publication status. We have created search strategy sample for MEDLINE, and have adapted similar search strategies for other electronic databases. Other resources We will also identify other sources, including conference abstracts and reference lists of included trials.

INPLASY registration number: This protocol was registered with the International Platform of Registered Systematic Review and Meta-Analysis Protocols (INPLASY) on 17 April 2020 and was last updated on 17 April 2020 (registration number INPLASY202040102). 
Comparator: All participants in the control group were healthy without NAFL and ACI.

Study designs to be included: All potential case-controlled studies (CCSs) will be included, which identified the association between NAFL and ACI.

Eligibility criteria: All potential casecontrolled studies (CCSs) will be included, which identified the association between NAFL and $\mathrm{ACl}$, regardless language and publication status limitations.

Information sources: Electronic searches This study will comprehensively search electronic databases (MEDLINE, EMBASE, Cochrane Library, Scopus, Web of Science, WANGFANG, and China National Knowledge Infrastructure) from their sources to the March 1, 2020 without limitations related to the language and publication status. We have created search strategy sample for MEDLINE, and have adapted similar search strategies for other electronic databases. Other resources We will also identify other sources, including conference abstracts and reference lists of included trials.

Main outcome(s): Outcomes are severity of $\mathrm{ACl}$ (measured by National Institutes of Health Stroke Scale, or other scales), serum levels of glucose, triglycerides, total cholesterol, low density lipoprotein cholesterol, high-density lipoprotein cholesterol, creatinine, serum alanine a minotransferase, as partate aminotransferase, and uric acid.

Data management: Two review authors will independently collect data from included articles. Any discrepancies between two review authors will be solved through discussion with a third review author. We will collect study title, first author, year of publication, location, participant characteristics (such as age, gender, severity of NAFL and $\mathrm{ACl}$ ), outcomes, results, conclusions, and conflict of interest.
Quality assessment / Risk of bias analysis: Study quality of included CCSs will be examined by two independent review authors using Newcastle-Ottawa Scale. Divisions between two review authors will be settled by discussion with a third review author.

Strategy of data synthesis: This study will employ RevMan V.5.3 to pool and analyze all extracted outcome data. We will exploit dichotomous data by risk ratio and $95 \%$ confidence intervals (Cls), and we will express continuous data by weighted mean difference or standardized mean difference and $95 \%$ Cls. We will use $I^{2}$ test to check heterogeneity. The values of $I^{2}$ are defined as follows: $I^{2} \leq 50 \%$ shows homogeneity, we will use a fixed-effects model, while $I^{2}>$ $\mathbf{5 0} \%$ suggests apparent heterogeneity, and we will utilize a random-effects model. We will carry out a meta-analysis if homogeneity is examined. Otherwise, we will perform a subgroup analysis to investigate the possible sources of distinct heterogeneity.

Subgroup analysis: If possible, a subgroup analysis will be performed according to the study characteristics, study quality, and outcomes.

Sensibility analysis: We will also carry out a sensitivity analysis to check the robustness of study results by removing the low quality studies.

Country(ies) involved: China.

Keywords: Acute cerebral infarction; nonalcoholic fatty liver; association. 